\title{
Left Ventricular Outflow Tract Obstruction Due to Anomalous Insertion of Papillary Muscle
}

\author{
Tetsuya Nomura, MD; Yoshiaki Harada, MD; Yoko Suzaki, MD; Hironori Hayashi, MD; \\ Hiroyuki Tanaka, MD; Jun Shiraishi, MD; Sumio Komatsu, MD; Yasuo Hosomi, MD; \\ Shinji Hirano, MD; Hitoshi Yaku, MD*; Nobuo Kitamura, MD*
}

\begin{abstract}
A 56-year-old man who complained of quadrantic hemianopsia was admitted to determine its etiology. Cerebral angiography revealed no organic stenosis. Echocardiography showed clear direct continuity between a hypertrophied anterolateral papillary muscle and the anterior mitral leaflet, and the left ventricular (LV) outflow tract (LVOT) was narrowed by the presence of an accessory papillary muscle. The LVOT obstruction caused an intra-LV pressure overload that resulted in LV concentric hypertrophy. Arrhythmia, such as paroxysmal atrial fibrillation (PAF), was thought to have caused a cerebral embolism. Mitral valve replacement (MVR), septal myectomy, and myectomy of the abnormal papillary muscle were performed, and complete release of the LVOT obstruction was accomplished. Anomalous insertion of papillary muscle is a rare cause of LVOT obstruction. Echocardiography was useful in identifying the papillary muscle malformation, and surgery was completely curative. (Circ J 2004; 68: 1219-1222)
\end{abstract}

Key Words: Anomalous insertion; Echocardiography; Left ventricular outflow tract obstruction; Papillary muscle

A 56-year-old man was admitted for investigation of quadrantic hemianopsia. He complained of general fatigue on slight effort and had experienced recurrent syncopal episodes during the previous 5 years. Cardiac hypertrophy had been diagnosed by echocardiography at another hospital 2 years before, and he was taking candesartan $8 \mathrm{mg} /$ day, atenolol $25 \mathrm{mg} /$ day, and cibenzoline $150 \mathrm{mg} /$ day. He did not have a family history of cardiovascular disease.

He suddenly developed left-upper quadrantic hemianopsia at rest in November 2001. Brain MRI revealed localized infarction in the right occipital lobe. No organic stenosis was found on cerebral angiography, and cardiogenic cerebral embolism was suspected. Blood pressure was $112 / 72 \mathrm{mmHg}$, and a grade $3 / 6$ harsh ejection systolic murmur was audible at the left sternal border in the third intercostal space. A 12-lead electrocardiogram showed sinus rhythm with left ventricular (LV) high voltage. Anteroposterior chest radiography indicated cardiomegaly (cardiothoracic ratio $=55 \%$ ), but there was no evidence of pulmonary congestion or pleural effusion. Transthoracic and transesophageal echocardiography showed intact aortic and mitral leaflets with mild aortic regurgitation and mitral regurgitation. The subaortic area was mildly narrowed by inward displacement of the ventricular septum. Definite direct continuity between the hypertrophied anterolateral papillary muscle and the anterior mitral leaflet was observed, and on the apical 4-chamber view the LV outflow

(Received May 8, 2003; revised manuscript received November 4, 2003; accepted November 7, 2003)

Department of Cardiology, Maizuru Medical Center, Yukinaga, Maizuru and $*$ Department of Cardiovascular Surgery, Kyoto Prefectural University of Medicine, Kyoto, Japan

Mailing address: Tetsuya Nomura, MD, Translational Research Center, Kyoto University Hospital, Kawahara-cho, Shogoin, Sakyo-ku, Kyoto 602-8507, Japan. E-mail: prinom@eos.ocn.ne.jp tract (LVOT) was narrowed by the presence of an accessory papillary muscle (Fig 1). Systolic anterior motion (SAM) of the anterior mitral leaflet could also be seen. Two-dimensional echocardiography revealed diffuse concentric hypertrophy, and the thickness of the septal and posterior walls was $17 \mathrm{~mm}$ and $15 \mathrm{~mm}$, respectively. The pressure gradient through the LVOT measured by continuous Doppler echocardiography was greater than $100 \mathrm{mmHg}$. Cardiac catheterization was performed, and coronary angiography confirmed the absence of coronary artery disease. Simultaneous measurement of the LV apical pressure and aortic pressure showed a $160 \mathrm{mmHg}$ pressure gradient, despite optimal medical therapy. There was no pressure gradient through the aortic valve. The post-extrasystolic contraction LV apical pressure was measured $346 \mathrm{mmHg}$ (Fig 5). Left ventriculography was not performed because of the high pressure and suspicion of a mural thrombus in the left ventricle. Finally, myocardial biopsy of the right ventricular (RV) septum was performed. Cardiac MRI showed diffuse LV hypertrophy and a hypertrophied anterolateral papillary muscle originating from the anterolateral wall of the left ventricle (Fig 2). ${ }^{201} \mathrm{Tl}$ and ${ }^{123}$ I-betamethylp-iodophenyl pentadecanoic acid myocardial scintigraphy at rest revealed diffuse hypertrophy. The results of every examination performed led us to conclude that the LVOT pressure gradient was caused by the anomalous hypertrophied papillary muscle, not by the asymmetrical hypertrophy of obstructive hypertrophic cardiomyopathy (HCM). The literature on this subject showed that resection of the abnormal papillary muscle and mitral valve replacement (MVR) would reduce the LVOT pressure gradient.

The operation was performed via a median sternotomy, with cardiopulmonary bypass and cardioplegic arrest. Upon opening the left atrium, the hypertrophied anterolateral papillary muscle was found to be directly attached to the anterolateral aspect of the middle of the anterior leaflet. 

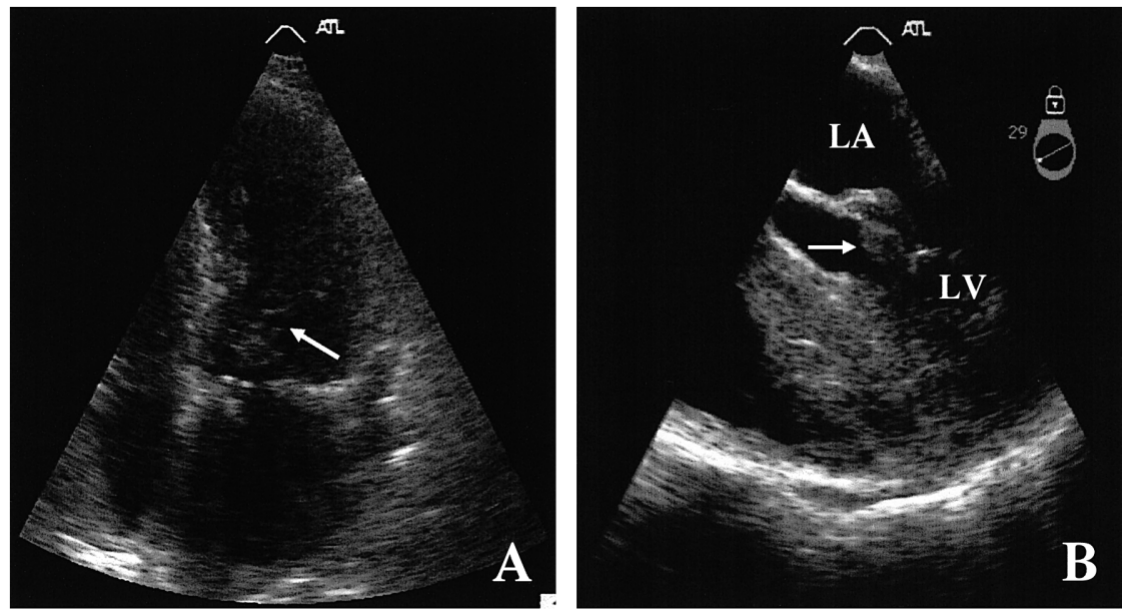

Fig 1. Transthoracic (A: apical 4-chamber approach) and transesophageal (B: upper gastric approach) echocardiography showing clear direct continuity between the hypertrophied anterolateral papillary muscle (arrows) and anterior mitral leaflet. LA, left atrium; LV, left ventricle.
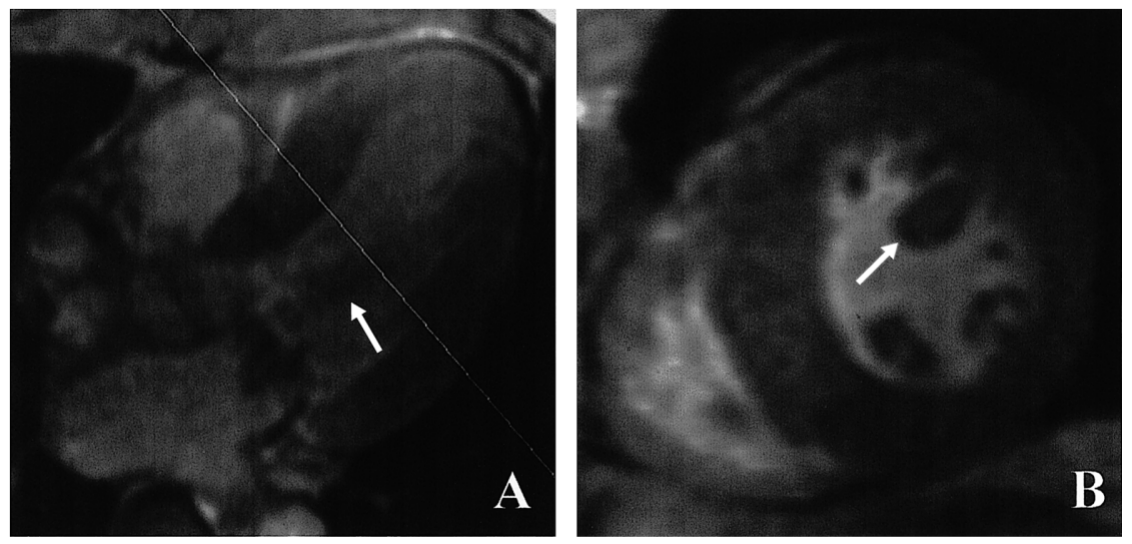

Fig 2. Cardiac MRI (A: long-axis view, B: short-axis view) showing diffuse LV hypertrophy and anomalous insertion of hypertrophied anterolateral papillary muscle (arrows).

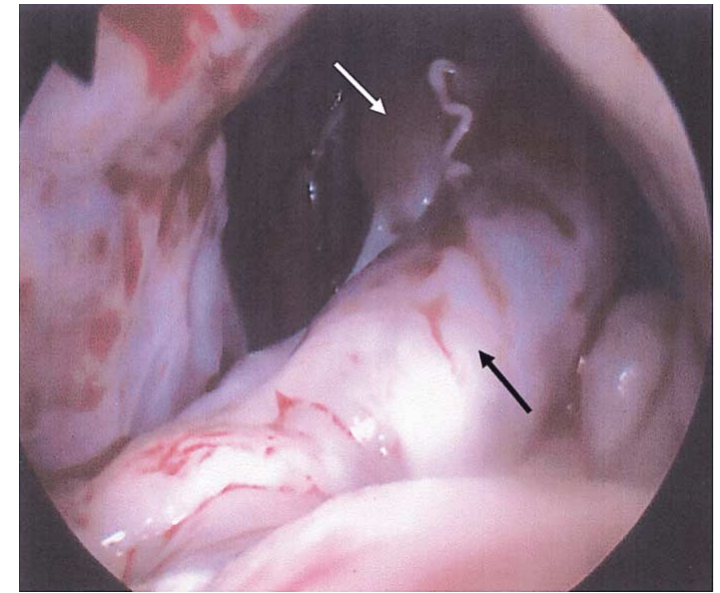

Fig 3. Intra-operative findings. An abnormally hypertrophied papillary muscle (black arrow) can be seen through the aortic valve. White arrow indicates the normal anterolateral papillary muscle.

The mitral valve was almost normal. Opening the ascending aorta by an oblique incision revealed an abnormally hypertrophied papillary muscle in the form of an almost 1-cm wide columnar muscular band immediately below the aortic leaflets (Fig 3). The hypertrophied papillary muscle was resected together with the attached normal anterior leaflet in block via the left atrium (Fig 4), and the mitral valve was replaced with a mechanical one; the hypertrophied septum was partially resected along the aortic

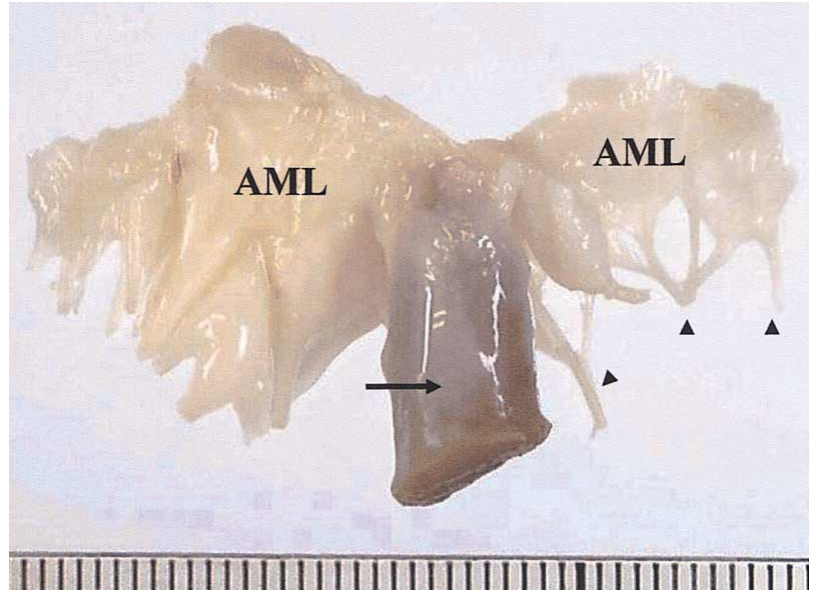

Fig 4. Gross specimen revealing direct attachment of the hypertrophied anterolateral papillary muscle (arrow) to the anterolateral aspect of the middle of the anterior leaflet. The mitral valve was almost normal. Arrowheads show the tendons to the normal anterolateral papillary muscle. AML, anterior mitral leaflet.

annulus. The patient was weaned from the cardiopulmonary bypass uneventfully with a small dose of cathecolaminergic agent.

Histologic examination of the both the resected septal myocardium and the biopsied RV endocardium showed irregular hypertrophy with irregular interstitial fibrosis and occasional vacuolar degeneration. The cardiac fibers were 

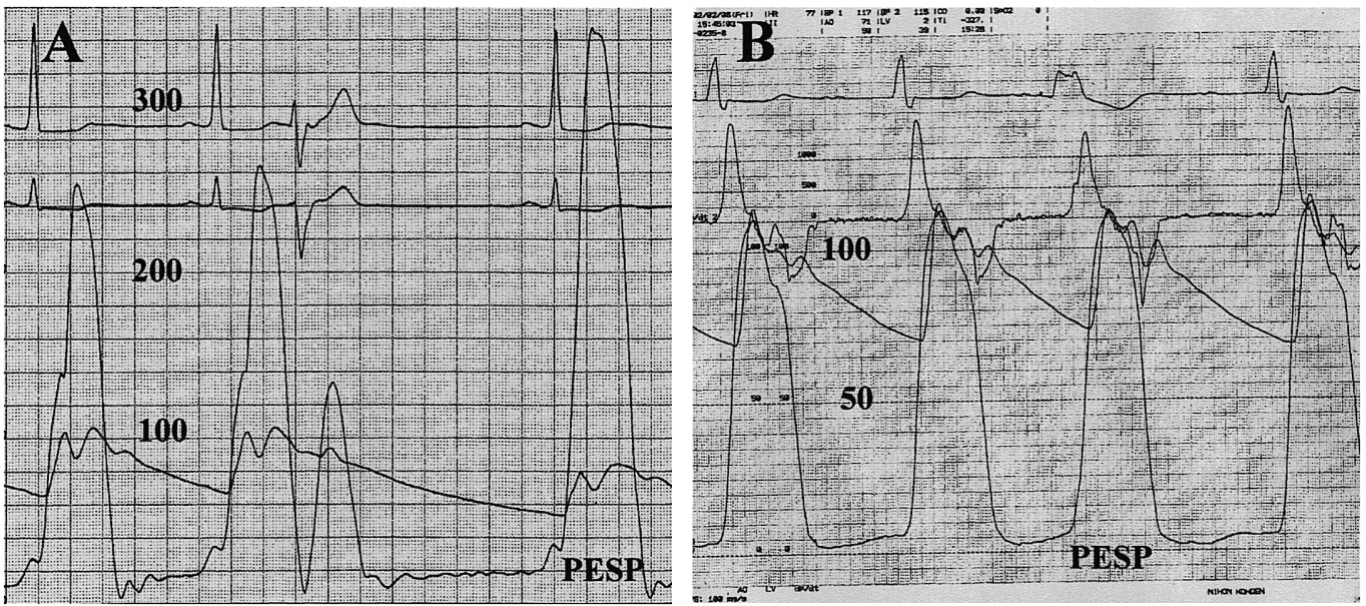

Fig 5. Simultaneous measurements of left ventricular apical pressure and aortic pressure shows a constant $160 \mathrm{mmHg}$ pressure gradient. Post-extrasystolic contraction left ventricular apical pressure measured $346 \mathrm{mmHg}$ (A: measurements in pre-operative period). Post-operative measurements showing complete disappearance of the pressure gradient through the LVOT (B). PESP, post-extrasystolic potentiation.

regularly arranged. No findings typical of HCM, such as disorganization and disarray of myocardial fibers and bizarre-shaped nuclei, were observed in the dissected fragment of myocardium, nor were there specific findings characteristic of rheumatic change or endocarditis in the valvular leaflets. In the postoperative period, he experienced paroxysmal atrial fibrillation (PAF) several times, which soon improved with intra-venous injections of antiarrhythmia drug. Follow-up echocardiography and cardiac angiography showed complete disappearance of the pressure gradient through the LVOT (Fig 5) and there have been no syncopal episodes during a follow-up period of 1 year.

\section{Discussion}

We have reported a case of anomalous insertion of papillary muscle that caused a severe pressure gradient between the LV apex and ascending aorta. Klues et al reported that malformations with direct continuity between a hypertrophied papillary muscle and mitral leaflet, as visualized by echocardiography, resulted in a long rigid area of midcavity narrowing that appeared to be solely or largely responsible for outflow obstruction!, 2 The hypertrophied papillary muscle obstructs the LVOT to such an extent that the Venturi effect, which was postulated by Roldan et $\mathrm{al}^{3}$ may easily be operating on the muscle well as on the anterior mitral leaflet. In the present patient, the outflow obstruction produced by the anomalous papillary muscle gradually induced LV hypertrophy, and after a long subclinical period, the obstruction became severe enough to cause syncopal attacks.

Most previous reports have related anomalous insertion of papillary muscle to obstructive HCM. Klues et al reported that 10 of $78(13 \%)$ mitral valve specimens from patients with obstructive HCM showed direct insertion of one or both LV papillary muscles into the anterior mitral leaflet; ${ }^{1}$ however, in our case, some results of examinations were not characteristic of HCM. For one thing, the LV hypertrophy was not typical. Of course we know that patients with hypertensive heart disease (HHD) sometimes show asymmetrical septal hypertrophy, but only $5 \%$ of patients with HCM show concentric hypertrophy ${ }^{4}$ and differentiation of HCM from HHD morphologically is often difficult5 For another, there were not the typical histologic findings of HCM, though the specificity is not so high, in either the resected septal myocardium or the biopsied RV endocardium. In any case, irrespective of HCM, we consider that the etiology of the LVOT obstruction was the anomalous insertion of the papillary muscle and SAM, and that intra-LV hypertension caused secondary concentric hypertrophy.

Fig 3 shows the abnormal hypertrophied papillary muscle band situated obliquely across the LVOT. We believe that the abnormal hypertrophied papillary muscle mass effect had a worse effect than would be expected from the extent of actual reduction of the LVOT area. Asymmetrical septal hypertrophy was not seen in this patient, but other unique malformations included anterior and inward displacement of the hypertrophied papillary muscles and the mitral leaflets coaptation point's anterior shift. These structural changes caused the onset of SAM by inducing the elongation of the anterior mitral leaflet. In addition, the Venturi effect, based on increased flow velocity in a narrowed outflow tract, will enhance and maintain SAM. Complex mechanisms would cause the initiation and resolution of SAM. Furthermore, as mentioned earlier, the Venturi effect may directly work on the abnormal papillary muscle. We think the mechanisms worked interdependently to produce the severe LVOT pressure gradient, but because abnormal papillary muscle was a primary factor in all the mechanisms, resecting it effectively released the LVOT pressure gradient.

The patient first complained of quadrantic hemianopsia, but no organic lesions were discovered in the cerebral arteries. Syncopal episodes in patients with obstructive HCM are frequently said to be related to atrial or ventricular tachyarrhythmias. In the present case, tachyarrhythmias, such as $\mathrm{PAF}$, and secondary cardiogenic thromboembolism are thought to have occurred.

During the surgical treatment of uncomplicated obstructive HCM, the obstruction is usually reduced by myectomy of the ventricular septum ${ }^{6}$ (Morrow procedure), but in cases such as the present one, to completely release the obstruction of the LVOT, mitral valve replacement and resection of the anomalous papillary muscle have been required, 
together with septal myectomy. Klues et al reported 2 cases treated by septal myectomy only in which there were persistent symptoms and incomplete relief of the obstruction (gradients of $60 \mathrm{mmHg}$ and $70 \mathrm{mmHg}$ ) because of continued midcavity apposition of the papillary muscle and ventricular septum! A novel operative approach for young patients in whom MVR is undesirable, was reported by Maron et $\mathrm{al}^{7}$ in which extending the muscle resection well beyond the site of the mid-ventricular obstruction is performed. Their method makes it possible to substantially widen middle LV cavity to the apical one, as well as the basal outflow tract. Resection of the hypertrophied papillary muscle, MVR and septal myectomy were performed in the present patient, and complete release of the LVOT obstruction was achieved. Occasionally, preoperative echocardiographic studies fail to identify this abnormality of the mitral apparatus, and the true anatomic continuity between the papillary muscle and mitral leaflet is visualized only when the cross-sectional plane is angulated slightly medially or laterally with respect to the center of the cavity. Whenever the absence of asymmetrical hypertrophy, the presence of a LVOT pressure gradient and an intact aortic valve are observed, the pathological and anatomical entity of anomalous insertion of papillary muscle should be considered in the differential diagnosis.

\section{References}

1. Klues HG, Roberts WC, Maron BJ. Anomalous insertion of papillary muscle directly into anterior miral leaflet in hypertrophic cardiomyopathy. Circulation 1991; 84: 1188-1197.

2. Klues HG, Maron BJ, Dollar AL, Roberts WC. Diversity of structural mitral valve alterations in hypertrophic cardiomyopathy. Circulation 1992; 85: $1651-1660$.

3. Roldan CA, Gurule FT, Shively BK. Anomalous papillary muscle producing dynamic left ventricular outflow tract obstruction. $J$ Am Soc Echocardiogr 1991; 4: 267-270.

4. Douglas WE, Harry R, Brian PK, William GW. Hypertrophic cardiomyopathy: Clinical spectrum and treatment. Circulation 1995; 92: $1680-1692$.

5. Tomoki N, Hiroki S, Noriyuki K, Satoshi Y, Akihiro A, Masao N. Can serum carnitine levels distinguish hypertrophic cardiomyopathy from hypertensive hearts? Hypertension 2000; 36: 215-219.

6. Morrow AG, Reitz BA, Epstein SE, Henry WL, Conkle DM, Itscoitz $\mathrm{SB}$, et al. Operative treatment in hypertrophic subaortic stenosis: Techniques and the results of pre-and post-operative assessments in 83 patients. Circulation 1975; 52: $88-102$.

7. Maron BJ, Nishimura RA, Danielson GK. Pitfalls in clinical recognition and a novel operative approach for hypertrophic cardiomyopathy with severe outflow obstruction due to anomalous papillary muscle. Circulation 1998; 98: 2505-2508. 